\title{
Measurements of the capture cross sections of natural silver in the resonance range with the time of flight technique
}

\author{
L. Šalamon ${ }^{1,2, a}$, B. Geslot ${ }^{1}$, J. Heyse ${ }^{2}$, S. Kopecky ${ }^{2}$, P. Leconte ${ }^{1}$, G. Noguere ${ }^{1}$, C. Paradela ${ }^{2}$, P. Schillebeeckx², \\ and L. Snoj ${ }^{3}$ \\ 1 DER, DEN, CEA, Cadarache, 13108 Saint-Paul-lez-Durance, France \\ 2 EC-JRC-Geel, 2440 Geel, Belgium \\ ${ }^{3}$ Reactor Physics Division, Jozef Stefan Institute, 1000 Ljubljana, Slovenia
}

\begin{abstract}
Neutron capture cross section measurements have been performed at the time-of-flight facility GELINA of the EC-JRC-Geel. Prompt gamma rays, originating from a natural silver sample, were detected by a pair of $\mathrm{C}_{6} \mathrm{D}_{6}$ liquid scintillation detectors. The total energy detection principle in combination with the pulse height weighting technique has been used. In this contribution the experimental details together with the data reduction process are described. In addition, first results of calculations with REFIT are presented to verify the quality of recommended cross section data in the resolved resonance region.
\end{abstract}

\section{Introduction}

Natural silver (composed of ${ }^{107} \mathrm{Ag}$ and ${ }^{109} \mathrm{Ag}$ ) is often used in an alloy together with cadmium and indium to produce control rods for water moderated power reactors. Such an alloy, referred to as AIC, provides good mechanical properties and results in an uniform absorption spectrum. Recently an evaluation of the resonance parameters for cadmium isotopes was performed by Volev et al. [1]. This evaluation, which was strongly based on results of experiment at the GELINA (Geel LINear Accelerator [2]) facility of EC-JRC-Geel, was included in the JEFF3.2 neutron library. In this contribution experiments to improve the nuclear data for ${ }^{107} \mathrm{Ag}$ and ${ }^{109} \mathrm{Ag}$ are described. The results of these experiments will be used to produce new evaluated data for ${ }^{107} \mathrm{Ag}$ and ${ }^{109} \mathrm{Ag}$ in the resonance region. This should result in a better prediction of the reactivity worth of AIC control rods.

\section{Experiment}

Capture experiments using natural silver samples were performed at GELINA facility of the JRC at Geel. GELINA is a time-of-flight facility providing a pulsed white neutron source. The electron linac accelerates pulsed electron beams up to $150 \mathrm{MeV}$ with repetition rates between 50 and $800 \mathrm{~Hz}$. At the exit of the linac, a compression magnet provides electron bursts with a width less than $1 \mathrm{~ns}$ [3]. The electrons are stopped in a mercury-cooled rotating uranium target and generate Bremsstrahlung radiation. Subsequently neutrons are produced via $(\gamma, \mathrm{n})$ and to lesser extent via $(\gamma, \mathrm{f})$ process [4]. To enhance the neutron spectra intensity at lower energies, two water-filled beryllium containers of $4 \mathrm{~cm}$ thickness below and above the uranium target are used as neutron moderators. The production rate of

a e-mail: linosalamon@hotmail.com neutrons is constantly monitored by $\mathrm{BF}_{3}$ proportional counters, placed at different places inside the neutron target hall. They are used for checking the stability of the accelerator and to normalize TOF spectra to the same neutron intensity.

The measurements were performed at flight path 5, at a distance of around $12.9 \mathrm{~m}$ from the neutron production target (Fig. 1). Shadow bars of $\mathrm{Cu}$ and $\mathrm{Pb}$ were placed close to the uranium target to reduce the intensity of the $\gamma$-ray flash and the fast neutron part of the spectra. The neutron beam was collimated to the diameter of around $75 \mathrm{~mm}$ at the sample position. The accelerator was operated in $800 \mathrm{~Hz}$ mode with a ${ }^{10} \mathrm{~B}$ overlap filter. Fixed $\mathrm{Na}$ and $\mathrm{S}$ black resonance filters were used for continuous monitoring the background at $2.85 \mathrm{keV}$ and $102 \mathrm{keV}$.

Due to the presence of the $\mathrm{S}$ filter no additional filter was needed to reduce the $\gamma$-ray flash. The measurement station was equipped with an air-conditioning system to keep the sample at a constant temperature of around $19^{\circ} \mathrm{C}$ and thus to minimize drift of the electronics. Prompt photons originating from the sample after a capture event were detected with a pair of $\mathrm{C}_{6} \mathrm{D}_{6}$ liquid scintillation detectors (NE230). The detectors formed an angle of $125^{\circ}$ with respect to the direction of the incoming neutron beam in order to minimise the effects of the anisotropy of the dipole primary radiation. Each scintillator was coupled with a boron-free quartz windowed EMI9823KQB photomultiplier (PMT). The anode signal of the PMT determined the arrival time of the neutron and the signal of the $9^{\text {th }}$ dynode was used to determine the energy deposited in the detector by the $\gamma$-rays. The discrimination level of the capture detection system was set to around $240 \mathrm{keV}$ deposited energy. The total energy detection principle in combination with the pulse height weighting technique [5] was used to make the efficiency to detect a capture event directly proportional to the total $\gamma$-ray energy deposited in the detector. The weighting function was determined 


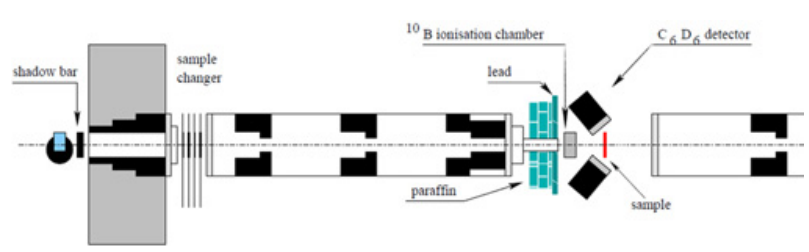

Figure 1. A schematic representation of the GELINA capture setup at flight path 5 .

for the mono-energetic $\gamma$-rays, which were assumed to be homogeneously distributed inside the sample. The detector response was calculated with the Monte Carlo simulations.

The incident neutron flux shape was measured in parallel using a ${ }^{10} \mathrm{~B}$ Frisch gridded ionisation chamber, placed at about $1 \mathrm{~m}$ before the sample. The detector consists of two ionization chambers with a common cathode loaded with two ${ }^{10} \mathrm{~B}$ layers with an areal density of $40 \mu \mathrm{g} / \mathrm{cm}^{2} .{ }^{10} \mathrm{~B}$ is evaporated back to back on a $30 \mu \mathrm{m}$ thick aluminium backing and serves as a neutron converter. The ${ }^{10} \mathrm{~B}(\mathrm{n}, \alpha)$ reaction was used to determine the energy dependence of the neutron flux.

The TOF $t_{m}$ of the registered neutron was derived from the time difference between the stop $\mathrm{T}_{\mathrm{s}}$ signal from the $\mathrm{C}_{6} \mathrm{D}_{6}$ detectors $/{ }^{10} \mathrm{~B}$-ionisation chamber and the start signal $\mathrm{T}_{0}$ of each electron pulse with $\mathrm{at}_{0}$ as the time offset as $\mathrm{t}_{\mathrm{m}}=\left(\mathrm{T}_{\mathrm{s}}-\mathrm{T}_{0}\right)+\mathrm{t}_{0}$. The time-of-flight and the amplitude of each event were recorded in the list mode using the data acquisition system developed at EC-JRC-Geel [6]. Data were stored in cycles of $900 \mathrm{~s}$. Only cycles for which the operating frequency and for which the total neutron intensity and response of the detectors deviated from the average by less than $0.05 \%, 2.5 \%$ and $3 \%$ respectively, were selected.

Three metallic discs of natural silver with different thicknesses $(0.25 \mathrm{~mm}, 0.126 \mathrm{~mm}$ and $0.06 \mathrm{~mm})$ were used for measurements. The area density was derived from a measurement of the weight and the effective area. The latter was deduced from a non-contact optical surface inspection with a microscopy-based system. Apart from the silver samples, additional measurements with only the sample holder and with the ${ }^{208} \mathrm{~Pb}$ in the beam were performed to determine the background contributions from the frame holder and scattered neutrons. A metallic ${ }^{197} \mathrm{Au}$ disc of $0.5 \mathrm{~mm}$ thickness was used to verify the normalization factor.

\section{Data reduction and analysis}

The experimental yield $Y_{\exp }$ was deduced from the ratio of the weighted response of the capture detectors and response of the flux detector as [7]:

$$
Y_{\exp }=\frac{\mathrm{N}_{\mathrm{c}}}{\varepsilon} \frac{\mathrm{C}_{\mathrm{w}}-B_{W}}{C_{\Phi-B_{\Phi}}} \frac{Y_{\Phi}}{T_{\Phi}} .
$$

The dead time corrected and weighted response of the $\mathrm{C}_{6} \mathrm{D}_{6}$ detectors is denoted by $\mathrm{C}_{\mathrm{w}}$ and the dead time corrected response of the flux detector by $\mathrm{C}_{\Phi}$. Their background contributions are $\mathrm{B}_{\mathrm{w}}$ and $\mathrm{B}_{\Phi}$, respectively. $\mathrm{N}_{\mathrm{c}}$ is the normalization factor and $\varepsilon$ is the efficiency to detect a capture event. The normalization factor merges together energy independent contributions such as the absolute neutron flux, the effective target area seen by the neutron beam, the efficiency of the flux detector and the solid angle subtended by the target and the $\mathrm{C}_{6} \mathrm{D}_{6}$ detectors [7]. The efficiency to detect a capture event depends on the neutron separation energy and the energy of the incoming neutron [7]. $\mathrm{Y}_{\Phi}$ and $\mathrm{T}_{\Phi}$ are the theoretical reaction yield of the boron ionization chamber and attenuation of the flux in the chamber respectively.

To derive the capture yield from the TOF spectra, the AGS (Analysis of Geel Spectra) package [8] was used. The package includes the most important data reduction procedures, such as dead time correction and background subtraction. It fully propagates both correlated and uncorrelated uncertainties, starting from the uncorrelated uncertainties due to counting statistics.

\subsection{Background correction for the flux response}

The background contribution for the neutron flux measurements was determined by applying the black resonance technique. The background was approximated by an analytical expression [7]:

$$
B_{\Phi}(t)=K_{\Phi}\left[a_{0}+a_{1} e^{-\lambda_{1} t}+a_{2} e^{-\lambda_{2}\left(t+t_{0}\right)}\right]
$$

consisting of a sum of a time independent component $a_{0}$ a time dependent contribution $a_{1} e^{-\lambda_{1} t}$ due to the neutrons scattered inside the detector station and neutrons scattered at other flight paths, and a term $a_{2} e^{-\lambda_{2}\left(t+t_{0}\right)}$ oming from the slow neutrons from previous accelerator pulses. The decay constant $\lambda_{1}$ as derived from the measurements with additional filters (Ag,W,Co), while $\lambda_{2}$ was obtained by extrapolating the TOF spectra at the end of the spectrum, i.e., for $t_{m}>8 \times 10^{5} \mathrm{~ns}$. The parameter $t_{0}$ is related to the operating frequency of accelerator $\left(\mathrm{t}_{0}=1.25 \mathrm{~ms}\right.$ for $800 \mathrm{~Hz}$ ). The amplitudes $a_{1}$ nd $a_{2}$ ere adjusted to the black resonance dips due to the presence of the $\mathrm{Na}$ and $\mathrm{S}$ filters. The factor $K_{\Phi}=1.00 \pm 0.03$ introduces a $3 \%$ correlated uncertainty to account for the uncertainties related to the background. Its uncertainty was derived from statistical analysis of the difference between the black resonance dips and the model given by Eq. (2). The response of the ionization chamber and its background contributions are presented in Fig. 2.

\subsection{Background correction for $C_{6} D_{6}$ response}

The total background for weighted response of the $\mathrm{C}_{6} \mathrm{D}_{6}$ detectors was described as [7]:

$$
\mathrm{B}_{\mathrm{w}}(\mathrm{t})=\mathrm{b}_{0}+\mathrm{k}_{1} \mathrm{C}_{\mathrm{w}, 0}(\mathrm{t})+\mathrm{k}_{2} \mathrm{R}_{\mathrm{n}}(\mathrm{t})\left[\mathrm{C}_{\mathrm{w}, \mathrm{Pb}}(\mathrm{t})-\mathrm{C}_{\mathrm{w}, 0}(\mathrm{t})\right],
$$

where $b_{0}$ is a time independent contribution coming from the ambient radiation and possible radioactivity in the sample and its surroundings, $\mathrm{C}_{\mathrm{w}, 0}(\mathrm{t})$ and $\mathrm{C}_{\mathrm{w}, \mathrm{Pb}}(\mathrm{t})$ are weighted spectra of the measurements with only a sample holder and with a $0.5 \mathrm{~mm}$ thick ${ }^{208} \mathrm{~Pb}$ sample in the neutron beam, respectively. They are both corrected for the time independent background. Weighting functions used to derive $\mathrm{C}_{\mathrm{w}, 0}(\mathrm{t})$ and $\mathrm{C}_{\mathrm{w}, \mathrm{Pb}}(\mathrm{t})$ are the same as the ones for the silver samples. $R_{n}(t)$ is the ratio of the neutron scattering yields of the natural $\mathrm{Ag}$ and ${ }^{208} \mathrm{~Pb}$ samples. This ratio was derived from the calculation of the ratio of the theoretical scattering yield of natural $\mathrm{Ag}$ to the one of 


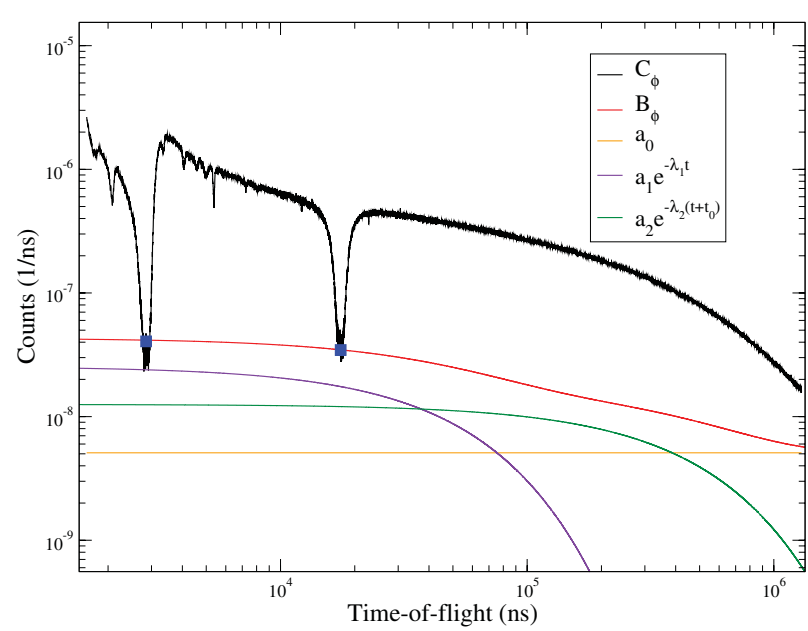

Figure 2. TOF spectra from the ionization chamber $\left(\mathrm{C}_{\Phi}\right)$ together with the total background $\mathrm{B}_{\Phi}$ and its components.

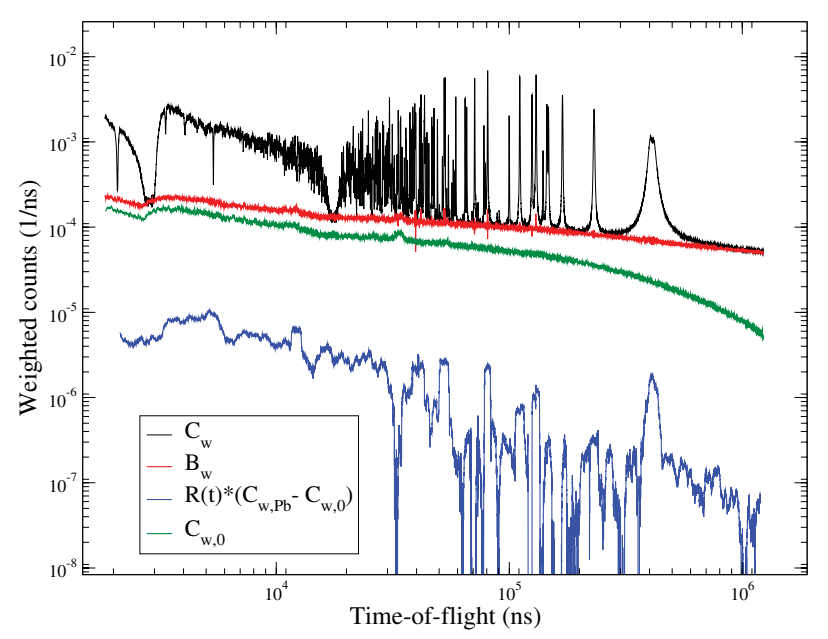

Figure 3. The weighted response of $\mathrm{C}_{6} \mathrm{D}_{6}$ detectors as a function of TOF for $0.25 \mathrm{~mm}$ thick $\mathrm{Ag}$ sample and the determined background with its different components.

${ }^{208} \mathrm{~Pb}$. Factors $\mathrm{k}_{1}=1 \pm 0.03$ and $\mathrm{k}_{2}=1 \pm 0.05$ account for the uncertainties due to the systematic effects in the background model. These uncertainties are based on a comparison of the background model given by Eq. (3) and the background dips present in TOF spectra due to the fixed black resonance filters. The weighted response of $\mathrm{C}_{6} \mathrm{D}_{6}$ detectors for a $0.25 \mathrm{~mm}$ thick Ag sample and its background contributions are shown in Fig. 3.

\subsection{Resonance analysis with REFIT}

The resonance shape analysis code REFIT [9] was used to calculate the theoretical capture yield. REFIT is based on the Reich-Moore approximation of the R-matrix theory and is using a least square method to obtain the resonance parameters. It accounts for various experimental effects [10] like neutron self-shielding, multiple interaction events, gamma-ray attenuation, Doppler broadening and response function of TOF spectrometer. Numerical response functions were used in this work. These functions were obtained from Monte Carlo simulations that have been validated by experiments [11]. They

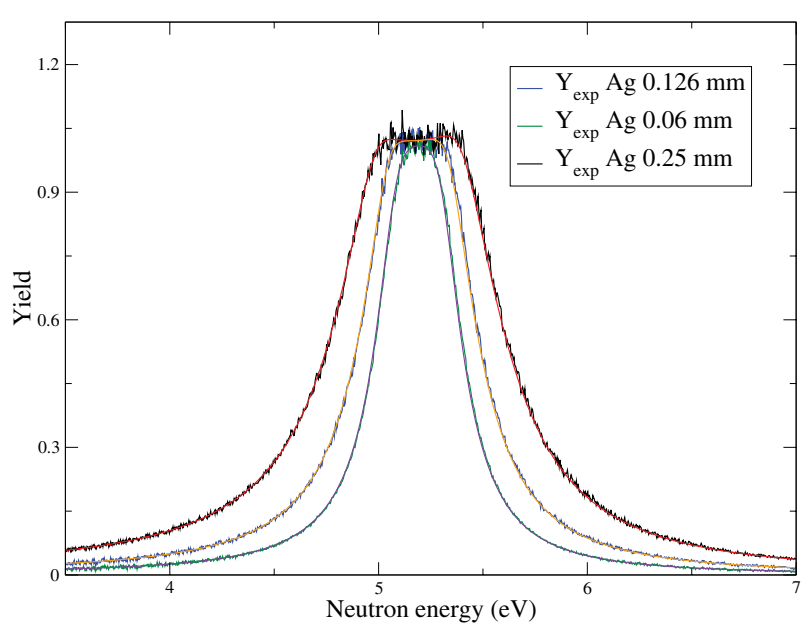

Figure 4. Results of a fit to the data using REFIT. The saturated resonance profile of the $5.2 \mathrm{eV}$ resonance of ${ }^{109} \mathrm{Ag}$ was used to determine the normalization factor.

Table 1. Normalization factors deduced from the capture measurements of silver samples relative to the one from the ${ }^{197} \mathrm{Au}$ sample.

\begin{tabular}{|c|c|}
\hline Sample & $\mathbf{N}_{\mathbf{c}, \mathbf{A g}} / \mathbf{N}_{\mathbf{c}, \mathbf{A u}}$ \\
\hline${ }^{197} \mathrm{Au} 0.5 \mathrm{~mm}$ & 1.000 \\
\hline $\mathrm{Ag} 0.25 \mathrm{~mm}$ & 1.006 \\
\hline $\mathrm{Ag} 0.126 \mathrm{~mm}$ & 1.013 \\
\hline $\mathrm{Ag} 0.06 \mathrm{~mm}$ & 1.014 \\
\hline
\end{tabular}

reflect mainly the neutron transport in the target-moderator assembly.

The flight path length of the capture station was determined from a fit to the ${ }^{197} \mathrm{Au}$ data in the energy region below $100 \mathrm{eV}$.

The experimental yields of the $0.25 \mathrm{~mm}$ and $0.126 \mathrm{~mm}$ thick Ag samples were internally normalized to the well isolated and saturated resonance at $5.2 \mathrm{eV}$ using the REFIT code. Internal normalization significantly reduces the correlated uncertainty as all systematic effects due to the position of the sample with respect to the neutron beam and detection system, and variations of the detector and accelerator conditions are eliminated. Only normalization factor was considered as a free parameter in the fit. Results of such a fit are shown in Fig. 4. For the $0.06 \mathrm{~mm}$ thick Ag sample, the $5.2 \mathrm{eV}$ resonance was not saturated. Normalization was obtained from the analysis of the $5.2 \mathrm{eV}$ resonance fixing the resonance parameters recommended in the JEFF-3.2 library. Normalization factors obtained from an analysis of the $5.2 \mathrm{eV}$ resonance profile were compared with the one derived from the $4.9 \mathrm{eV}$ saturated resonance profile of the ${ }^{197} \mathrm{Au}$ sample data. These normalization factors are in good agreement and their ratios are given in Table 1.

The final experimental capture yield obtained from measurements with the $0.25 \mathrm{~mm}$ thick $\mathrm{Ag}$ is shown in Fig. 5. This experimental yield is compared in Fig. 6 with the theoretical yield based on the resonance parameters of ${ }^{107} \mathrm{Ag}$ and ${ }^{109} \mathrm{Ag}$ recommended in the JEFF-3.2 library. As seen from the Fig. 6, there are discrepancies between experimental and the theoretical yield based on the JEFF3.2 parameters. 


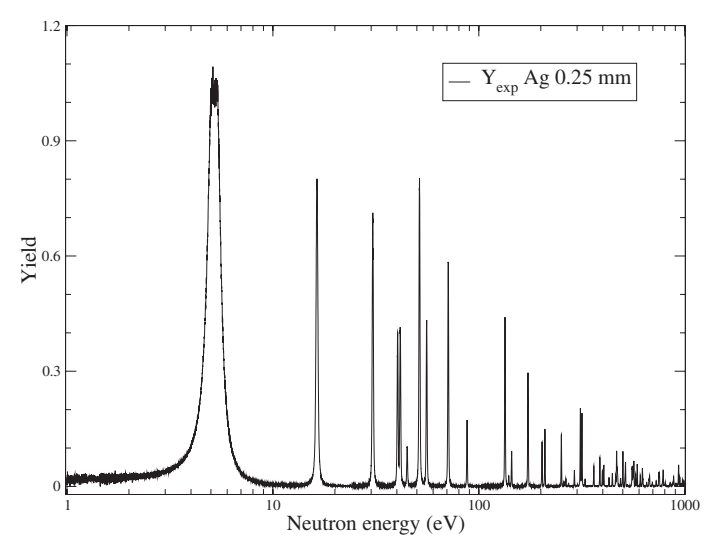

Figure 5. Final experimental capture yield of the $0.25 \mathrm{~mm}$ thick $\mathrm{Ag}$ sample for the incident neutron energy region up to $1 \mathrm{keV}$.

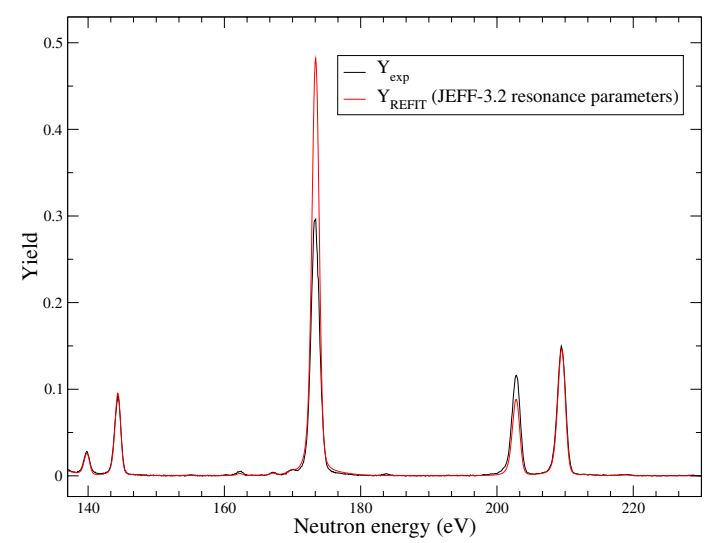

Figure 6. A comparison between final experimental capture yield and the REFIT calculation in the resolved resonance region, using JEFF-3.2 evaluated data.

\section{Conclusions}

This work presents the experimental details, basic steps in data reduction and analysis of neutron capture experiments on natural silver samples performed at the TOF facility GELINA. These experiments were performed to improve the resonance parameters for neutron induced reactions on ${ }^{107} \mathrm{Ag}$ and ${ }^{109} \mathrm{Ag}$. A comparison between the experimental and calculated capture yield reveals problems with some of the resonance parameters below $1 \mathrm{keV}$ that are recommended in the JEFF-3.2 library. Hence, a new evaluation including the data presented in this work is recommended.

Authors would like to thank technical staff for the decicated and skillful operation of GELINA. We are also grateful to scientists of EC-JRC-Geel for their valuable comments and suggestions. Šalamon L. acknowledges GENTLE project for the suport during his stay at EC-JRC-Geel.

\section{References}

[1] K. Volev et al., Nucl. Instr. Meth. Phys. Res. B 300, 11-29 (2013)

[2] W. Mondelaers, P. Schillebeeckx, Notizario 11, 19 (2006)

[3] D. Tronc, J.M. Salome, K.H. Böckhoff, Nucl. Instr. Meth. Phys. Res. A 228, 217 (1985)

[4] J.M. Salome, Nucl. Instr. Meth. 179, 13-19 (1981)

[5] A. Borella et al., Nucl. Instr. Meth. Phys. Res. A 577, 626-640 (2007)

[6] S. de Jonge, Int. Rep. G/DE/R/24/87, IRMM, Geel (1987)

[7] P. Schillebeeckx et al., Nucl. Data Sheets 113, 3054-3100 (2012)

[8] B. Becker et al., Jour. Instr. 7, P11002 (2007)

[9] M.C. Moxon, J.B. Brisland, AEA Techn. AEAInTec-0630 (1991)

[10] P. Schillebeeckx et al., J. Korean Phys. Soc. 59, 1563-1568 (2011)

[11] M. Flaska et al., Nucl. Instr. Meth. Phys. Res. A 531, 392 (2004) 